\title{
Exploring the use of Manual Liquid Based Cytology, Cell Block with Immunomarkers p16/ki67, VIA and HPV DNA Testing as a Strategy for Cervical Cancer Screening in LMIC
}

\author{
Nandini Nandish Manoli ${ }^{*}, 1,2$, Devananda Devegowda ${ }^{2}$, Ashoka Varshini ${ }^{1}$, Pushkal Sinduvadi Ramesh ${ }^{2}, \mathrm{Sherin}_{\mathrm{Susheel} \mathrm{Mathew}}{ }^{3}$, \\ Nandish Siddappa Manoli ${ }^{4}$
}

${ }^{1}$ Department of Pathology, JSS Medical College, JSS Academy of Higher Education \& Research, 570015, India.

${ }^{2}$ Centre of Excellence in Molecular Biology \& Regenerative Medicine, Department of Biochemistry, JSS Medical College, JSS Academy of Higher Education \& Research,570015, India.

${ }^{3}$ Department of Pathology, Dr Somervell Memorial Church of South India Medical College,695504, India

${ }^{4}$ Department of Obstetrics and Gynecology, JSS Medical College, JSS Academy of Higher Education \& Research, 570015, India

\begin{tabular}{l} 
A R T I C L E I N F O \\
\hline Article history: \\
Received: 05 September, 2018 \\
Revised: 30 September, 2018 \\
Accepted: 14 November, 2018 \\
\hline Keywords: \\
Manual Liquid Based Cytology \\
Cervix \\
Immunomarkers
\end{tabular}

\begin{tabular}{l} 
A B S T R A C T \\
\hline Cervical cancer is the 4th most common cancer in women in low to middle income group \\
countries (LMIC). Various methods for screening cervical cancer are practiced, such as \\
the Conventional Pap Smear (CPS), Liquid Based Cytology with its ancillary techniques \\
like Cell Block with immunocytochemistry. VIA is another method which is being advocated \\
as a primary screening tool. Molecular diagnostics such as use of HPV DNA testing has \\
been at the forefront of the screening programs. In the present study, we have utilized all \\
the above methods by using cost effective in-house procedures to explore their possible \\
utility in the clinical settings. We found them useful with need for more work and training \\
of personnel for better diagnosis of cervical cancer.
\end{tabular}

\section{Introduction}

Even though Cervical Cancer (CC) is the leading cause of death in women in the modern world, the incidence varies in developed and developing countries. India accounts for quarter of cervical cancer incidence in the world with 20.2 per 100,000 new cases of CC diagnosed and 11.1 per 100,000 deaths annually [1].

Screening by various methods can bring down the incidence of cervical cancer which has been successfully implemented in developed countries. But, the lack of infrastructure, national policies, poverty, lack of financial resources, inadequately trained health care providers, improper implementation of the screening programs with lack of education of women especially in the rural population has prevented the control of cervical cancer incidence in countries like India. This renders a huge load leading to a major global impact on health care of women [2, 3, 4].

Screening tests for cervical cancer include:

- Conventional exfoliative cervico-vaginal cytology i.e. the cervical (Pap) smear.

- Manual liquid-based cytology

*Corresponding Author: Nandini Nandish Manoli, Department of Pathology,JSS Medical College, JSSAHER, +919448978276 \& nandinimanoli65@gmail.com www.astesj.com

https://dx.doi.org/10.25046/aj030625
- Fluid sampling technics with automated thin layer preparation (liquid-based cytology)

- Cell block technique with immunomarker study

- HPV DNA testing

- Polar probe

- Laser induced fluorescence

- Visual inspection of cervix after applying Lugol's iodine (VILI) or acetic acid (VIA)

- Speculoscopy

- Cervicography

1.1. Exfoliative cytology (conventional Pap smear)

Conventional Pap smear has been the standard screening method for cervical cancer screening from the past several years. It includes sampling of material from the junction between the ecto- and endo-cervix using an Ayre's spatula. The material obtained is spread on to the clean glass slide which is stained by routine pap stain and studied by the Cytopathologist. It has limitations due to many errors either due to sampling (5-10\%), interpretation and obscuring factors like blood which hamper the 
accurate diagnosis. Only $20 \%$ of the sample taken gets spread on to the slide which hampers the sensitivity $[5,6]$.

\subsection{Manual liquid-based cytology}

Liquid based cytology is a technique wherein cells are arranged in a single or monolayer on a clean glass slide. It is a method which helps to remove obscuring factors seen in conventional method. There are two automated methods followed in developed countries: the SurePath and ThinPrep. These methods improve the sensitivity and specificity and also help in using the remaining material for ancillary techniques like cell block with IHC and HPV DNA testing. But these automated methods have their limitations as they are expensive to be used in LMIC.

To overcome these limitations, we have utilized an in-house cost-effective method of Manual Liquid Based Cytology (MLBC). We used simple machines like centrifuge and the sample collected in liquid fixative which was processed in a polymer solution prepared in the laboratory [7].

\subsection{HPV Testing}

Epidemiological studies and mechanistic evidence has led to the conclusion that $70 \%$ of cervical cancer cases are attributed to HPV-16 \& HPV-18, the high-risk subtypes of HPV. Cytology based cervical screening has led to the reduction in the incidence and mortality rates of cervical cancer and evidence suggests that inclusion of HPV testing could further refine the screening programs. Also, HPV testing has enormous potential to be used as a cost-effective primary screening module, to identify women with greater risk of disease progression and as a test of cure of disease $[3,8,9]$.

\subsection{Cell Block Technique}

Cell block technique is a method wherein the residual material in a sample can be processed to form a tissue block. The process employed can be varied from alcohol, formalin, agarose or thromboplastin to form a cell pellet which is processed like a tissue. The advantages are, multiple sections can be taken and can be used for immunocytochemistry (ICC) to improve the diagnosis of cervical lesions. Cell block can be used as histopathology tissue for controls in cytopathology laboratories. Only limitation is the time required for processing a cell block and the extra cost for the preparation [10].

\subsection{Visual inspection tests}

Visual inspection tests with 3-5\% acetic acid (VIA) and/or Lugol's iodine (VILI) appear to be a satisfactory alternative screening approach to cytology. These tests have been used since the 1990 s, mainly in poor resource settings. They are simple, costeffective with relative ease of use and may be performed by different healthcare workers (physicians, nurse, midwives and technicians). Moreover, this approach does not require high technology or infrastructure and has been shown to reduce mortality in developing countries $[8,11,12]$.

\section{Material and Methods}

This was a prospective study carried out from January 2017 to June 2018 in a tertiary care hospital of South India. The study was conducted after obtaining Institutional Ethical clearance from the committee. A total of 100 subjects within the age group of 20-70 years attending the OBG department with gynecological complaints were recruited for the study. Subjects with a history of abnormal Pap tests were included in the study and subjects who did not give consent to participate were excluded from the study. Samples were collected from the recruited subjects only after obtaining signed informed consent.

\subsection{Sample collection and processing}

Liquid based cervical cytology samples were collected using an endo-cervical cell collection device (Cervex-Brush $\AA$-Rovers medical devices). All the 100 samples were subjected to conventional Pap smear testing and manual liquid-based cytology analysis which was subjected to ancillary tests ieHPV testing and cell block processing. Due to the inadequacy of the sample for DNA extraction, only 68 cases were subjected to HPV DNA detection by Polymerase chain reaction. A total of 25 cases of cell block with HPV correlation were subjected to immunecytochemical analysis with p16 and ki67 markers.

\subsection{DNA extraction \& Polymerase Chain Reaction for HPV detection}

Samples are collected using cyto brush was transferred to a sterile capped container and immediately transported to the hospital's molecular laboratory. Samples were centrifuged at 10,000 rpm for $5 \mathrm{~min}$ to pellet down the cells and the cells were subjected to DNA extraction. DNA from the samples was extracted using HiPuraTM Multi-sample DNA purification kit (HiMedia, India) according to the manufacturer's instructions. PCR was performed using consensus MY09/MY11 primers that targets a 450bp region in L1 gene of the HPV. PCR was performed on Eppendorf's Mastercycler gradient as described in [13]. The positive DNA samples from the PCR was then subjected to type specific PCR with specific primers. Previously described primers described by [14] were utilized for the PCR experiments.

\section{3. p16and ki67 immunocytochemistry with cell blocks}

Immunocytochemistry was performed on the formalin-fixed and paraffin-embedded cell block sections by DAB chromogen method. Mouse monoclonal anti-p16 antibody was used. Scoring was done as following: Negative (no staining or $<3$ positively stained cells), $1+(3-10$ positively stained cells $), 2+(>10$ positively stained cells). Along with the cell number, staining intensity was also taken into consideration. For ki67 only nuclear staining with less than or more than 10 cells were taken as weak or strong positivity.

\subsection{Visual inspection with acetic acid (VIA)}

We conducted a study with a primary health centre for correlation of VIA with CPS on 100 cases at healthcare camps. The study took into consideration whether the VIA test was adequate or inadequate with visualization of the squamocolumnar junction or not. Pap smears with Ayre's spatula were taken by trained health workers at these camps. 5\% acetoacetic acid was applied to the cervix which was visualized by a Gynecologist or the health worker

\section{Results}

A total of 68 samples were subjected to HPV detection and genotyping by PCR in our study. Out of 68 cases, 12 were positive for HPV (8.16\%) and out of these $12 \mathrm{HPV}$ positive cases 7 were positive for high risk HPV type 16. None of the samples were HPV 
18 positive. Since we did not have positive controls for other high risk HPV subtypes, we could not test the status of these samples for other HPV subtypes.

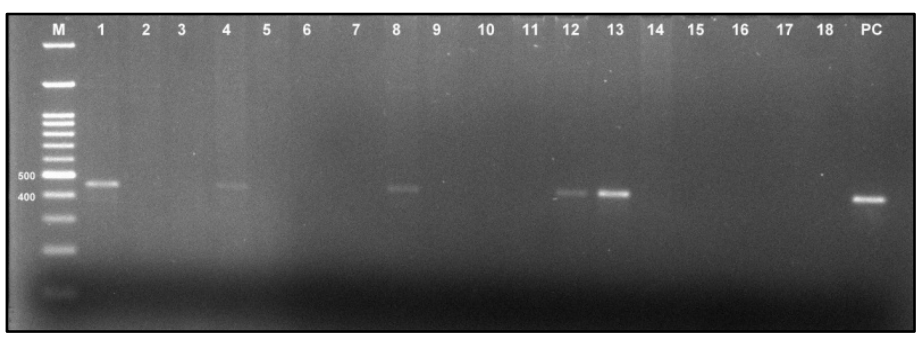

Figure 1: Representative gel image showing amplified HPV gene product of 450bp ( $\mathrm{M}=$ Marker, $\mathrm{PC}=$ Positive control)

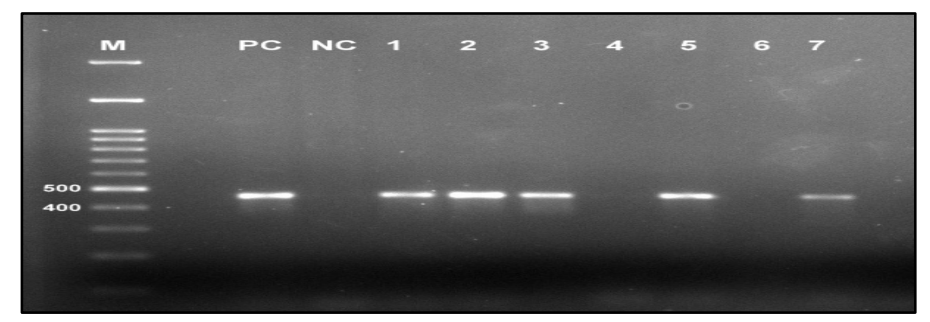

Figure 2: Representative gel image showing amplified HPV 16 gene product of 468bp ( $\mathrm{M}=$ Marker, $\mathrm{PC}=$ Positive control, $\mathrm{NC}=$ Negative control $)$

Twenty-five cases of cell block with HPV correlation showed 6 cases with HPV positivity. All 25 cases were subjected to p16 ink 4a IHC, of which 18 cases of chronic cervicitis were negative, two cases of koilocytic atypia were negative, one case of LSIL was weak positive, two cases of HSIL were strong positive and two cases of SCC were also strongly positive.

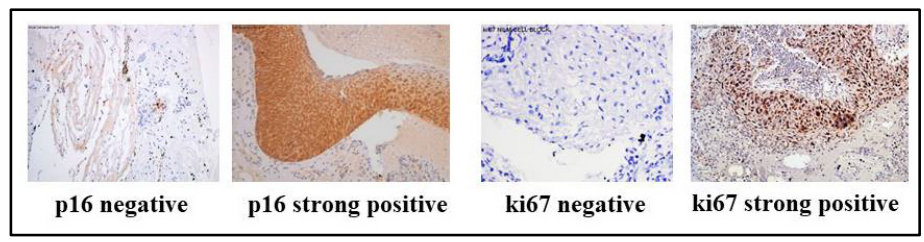

Figure 3: Representative images of IHC stained for p16 and ki67 immunomarkers

In cell blocks of NILM(negative) and squamous cell carcinoma(positive)

Table 1: Correlation of histopathological characteristics with HPV DNA positivity

\begin{tabular}{|l|l|l|l|l|}
\hline & $\begin{array}{l}\text { Hegative } \\
\text { HR- } \\
\text { HPV(16/18) }\end{array}$ & $\begin{array}{l}\text { Positive } \\
\text { HR- } \\
\text { HPV(16) }\end{array}$ & $\begin{array}{l}\text { HPV } \\
\text { Negative }\end{array}$ & \\
\hline $\begin{array}{l}\text { Chronic } \\
\text { Cervicitis }\end{array}$ & 1 & 0 & 6 & 07 \\
\hline LGSIL & 3 & 3 & 4 & 10 \\
\hline HGSIL & 0 & 1 & 6 & 07 \\
\hline SCC & 0 & 3 & 2 & 05 \\
\hline Total & 4 & 7 & 18 & 29 \\
\hline
\end{tabular}

Table 2: Correlation of cell block staining with HPV DNA positivity.

\begin{tabular}{|c|c|c|c|}
\hline & HPV & & \\
\hline \multirow{3}{*}{$\begin{array}{l}\text { P16 ON } \\
\text { CELL } \\
\text { BLOCK }\end{array}$} & & Positive & Negative \\
\hline & Positive & 5 & 0 \\
\hline & Negative & 1 & 19 \\
\hline
\end{tabular}

Statistical analysis

Correlation of HPV DNA Correlation of Cell block and testing with histopathology HPV DNA testing

Sensitivity: $45 \%$

Sensitivity: $83.3 \%$

Specificity: $85 \%$

Specificity: $100 \%$

PPV: $91 \%$

PPV : $100 \%$

NPV: $24 \%$

NPV: $95 \%$

Table 3: Correlation of histopathology with cell block and conventional Pap smear

\begin{tabular}{|l|l|l|l|l|}
\hline CELL BLOCK & $\begin{array}{l}\text { CHRONIC } \\
\text { CERVICTIS(10) }\end{array}$ & LSIL(14) & HSI(12) & SCC(7) \\
\hline NILM & $10(100 \%)$ & 0 & 0 & 0 \\
\hline $\begin{array}{l}\text { KOILOCYTIC } \\
\text { ATYPIA }\end{array}$ & 0 & $2(14 \%)$ & $2(17 \%)$ & 0 \\
\hline LSIL & 0 & $12(86 \%)$ & $1(8 \%)$ & 0 \\
\hline HSIL & 0 & 0 & $9(75 \%)$ & $1(14 \%)$ \\
\hline SCC & 0 & 0 & 0 & $6(86 \%)$ \\
\hline CPS & $10(100 \%)$ & $2(13 \%)$ & & $3(42 \%)$ \\
\hline NILM & & $8(53 \%)$ & $10(84 \%)$ & $1(16 \%)$ \\
\hline KOILOCYTIC \\
ATYPIA
\end{tabular}

Table 4: Correlation of VIA with conventional Pap smear

\begin{tabular}{|l|l|l|}
\hline Diagnosis & CPS & VIA \\
\hline NILM & 47 & 35 \\
\hline LSIL & 04 & 1 \\
\hline HSIL & 07 & 1 \\
\hline SCC & 12 & 2 \\
\hline Endo-cervical carcinoma in-situ & 05 & - \\
\hline ASCUS & 06 & 1 \\
\hline High grade carcinoma & 03 & 1 \\
\hline Unsatisfactory & 16 & 4 \\
\hline total & 100 & 47 \\
\hline
\end{tabular}

Of the 63 cases, 20 cases are without any correlation wherein 10 cases were VIA negative. Thus, the percentage of missed cases on VIA was $16 \%$.

\section{Discussion}

Cervical cancer screening and detection has improved from the days of conventional Pap smear screening to molecular tests in developed countries where government national health care policies have initiated screening programs leading to the reduction in the incidence. In developing country like ours, the screening programs have not got its wings due to various reasons. Attempts to make a low-cost method of early detection lead us to start an MLBC technique which reduces obscuring factors and spreads the cells in a monolayer for a clearer viewing of the cells. Similar technique has been used by many investigators as it has additional advantage of ancillary studies like testing for HPV DNA, preparation of cell block for immune-marker studies $[7,15,16]$.

HPV as a primary screening test has been advocated and being followed in European countries it has also found its feasibility in LMIC countries because of availability of many commercially available kits. They have become a milestone in the more effective 
screening of cervical cancer and prolonging the screening interval for patients.

We have standardized our own in-house HPV DNA testing methodology with a turnaround time of one day. The World Health Organization (WHO) recommends targeting HPVscreening to women who are 30 years of age and older because of their higher risk of $\mathrm{CC}$, and that priority should be given to screening women aged 30-49 years (WHO screening recommendation update 2014) [3].

\subsection{Triage of HPV-Positive Women}

HPV-based screening has a low positive predictive value for $\mathrm{CC}$ because it does not directly test forcancer, but for HPV infection instead. At the present time, three test methods can potentially be used as triage test: visual methods(VIA/VILLI); cytology; and molecular testing. To date, there is no clear evidence to determine which strategy should be prioritized. Therefore, the choice of test essentially depends on the available resource [12].

\subsection{Triage with cytology}

Cytology is the most widely recommended test to triage HPVpositive women, where quality-assured cytology is available. HPV-positive women with a cytology diagnosis of ASCUS or worse are referred for colposcopy, and the rest are advised to have repeat HPV testing after 1 year. Cytology performs better in a triaging scenario, since the prevalence of disease is high in the sample and cytologists have a limited number of specimens to evaluate. The current recommendations by the American Society for Colposcopy and Cervical Pathology (ASCCP) are direct referral to colposcopy for HPV $16 / 18$ positive women and repeat testing after 1 year for women positive for other HPV types [17].

\subsection{Triaging with Biomarkers}

LBC with immunocytochemistry and cell block sections with immunohistochemistry result in enhanced specimen quality, and accurate diagnosis, and diminished false negative cases. LBC has potential as a screening tool for cancer and precancerous lesions in several tissues other than gynecologic organs.

Cell block tissues made from remnants and residual LBC samples, aspirates, and fluid samples may also have applications for practice in the field of cytopathology. We have used a cost effective method by using MLBC in our set up to be used for HPV and cell block with p16 and ki67 as immune markers [18]. These markers are known to highlight the HSIL and squamous cell carcinoma cases of cervix. p16 can also diagnose LSIL cases even though it also gives positivity for endometrial cell tubal metaplasia and squamous metaplasia which will not be given positive by ki67 [15]. Thus, the use of p16inka4a and ki67 on cell blocks will enhance high grade/malignant lesions of the cervix from the non-neoplastic conditions and thus improve diagnostic accuracy as we found in our study.

\subsection{Triaging with VIA}

VIA which is a good approach for screening and treating in resource poor settings where cytology and HPV testing cannot be done is useful when the skill and knowledge about the technique is good. We found that it has its limitations as found by many workers [17].

\section{Conclusion}

Cervical cancer screening in low to middle income countries still needs to be refined in terms of affordability and accessibility. Current strategies for cervical cancer screening are not being implemented to its full potential mainly due to the lack of training, high cost and need of well set-up screening centers. There are various methods for screening cervical cancer in LMIC, which are being done in a small scale either in the form of research studies or by NGOs with whom we joined hands and did a study on VIA. In our study we explored the usage of multi-algorithm screening strategy for the screening of cervical cancer in a tertiary care hospital.

There is a need for a uniform policy of screening of women at the primary health care center level with increasing the awareness of the different methods among the public. Also, there is a need for well-trained health workers and Cytopathologists to diagnose and maintain follow up about cervical cancer with a cancer registry.

\section{References}

[1] J.Ferlay, I. Soerjomataram, R. Dikshit, S. Eser, C. Mathers, M. Rebelo, D.M Parkin, D. Forman, and F. Bray, "Cancer incidence and mortality worldwide: sources, methods and major patterns in GLOBOCAN 2012", INT J CANCER, 136(5), E359-E386,2015. https://doi.org/10.1002/ijc.29210

[2] K.S Tewari, A. Agarwal, A. Pathak, A. Ramesh, B. Parikh, M. Singhal, G. Saini, P.V Sushma, N. Huilgol, S. Gundeti and S. Gupta, "Meeting report : First Indian national conference on cervical cancer management-expert recommendations and identification of barriers to implementation", GYNECOLONCOLRESPRACT,5(5),2018. https://doi.org/10.1186/s40661018-0061-5

[3] R. Catarino, P. Petignat, G. Dongui, and P. Vassilakos, "Cervical cancer screening in developing countries at a crossroad: Emerging technologies and policy choices", WORLD J CLIN ONCOL, 6(6), 281, 2015. https://doi.org/10.5306/wjco.v6.i6.281

[4] S. Bobdey, J. Sathwara, A. Jain and G. Balasubramaniam, "Burden of cervical cancer and role of screening in India", INDIAN J MED PAEDIATR ONCOL, 37(4), 278, 2016. http://doi.org/10.4103/0971-5851.195751

[5] J.Sherris, "Cervical cancer in the developing world", WESTERN J MED, 175(4), 231-233, 2001. https://doi.org/10.1136/ewjm.175.4.231

[6] R.A Kerkar, and Y. V Kulkarni, "Screening for cervical cancer: an overview", J OBSTET GYNECOL INDIA, 56(2), 115-122, 2006.

[7] N.M Nandini, S.M Nandish, P. Pallavi, S.K Akshatha, A.P Chandrashekhar, S. Anjali, and M. Dhar, "Manual liquid based cytology in primary screening for cervical cancer-a cost effective preposition for scarce resource settings", ASIAN PAC J CANCER P, 13(8), 3645-3651, 2012. https://doi.org/10.7314/APJCP.2012.13.8.3645

[8] N. Wentzensen, M. Schiffman, T.Palmer, M.Arbyn, "Triage of HPV positive women in cervical cancer screening", J CLIN VIROL,76,49-55,2016. https://doi.org/10.1016/j.jcv.2015.11.015

[9] T.C Wright, M.H Stoler, C.M Behrens, A. Sharma, G. Zhang, T.L Wright, "Primary cervical cancer screening with human papillomavirus: end of study results from the ATHENA study using HPV as the first-line screening test", GYNECOLONCOL,136(2),189-197,2015. https://doi.org/10.1016/j.ygyno.2014.11.076

[10] L. Skoog, and E. Tani, "Immunocytochemistry: an indispensable technique in routine cytology", CYTOPATHOLOGY, 22(4), 215-229,2011. https://doi.org/10.1111/j.1365-2303.2011.00887.x

[11] L.Denny, M. Quinn and R. Sankaranarayanan, "Screening for cervical cancer in developing countries", VACCINE, 24, S71-S77,2006. https://doi.org/10.1016/j.vaccine.2006.05.121

[12] U.R Poli, P.D Bidinger, and S. Gowrishankar, "Visual inspection with acetic acid (via) screening program: 7 years experience in early detection of cervical cancer and pre-cancers in rural South India", INDIAN J COMMUNITY MED, 40(3), 203, 2015. https://doi.org/10.4103/0970-0218.158873

[13] P.S Ramesh, D. Devegowda, P.R Naik, P. Doddamani, And S.M Nataraj, "Evaluating the Feasibility of Nested PCR as a Screening Tool to Detect HPV Infection in Saliva of Oral Squamous Cell Carcinoma Subjects", 12(7),2018. https://doi.org/10.7860/JCDR/2018/34880.11806

[14] S. K Bandhary, V.Shetty, M.Saldanha, P.Gatti, D. Devegowda, S.R Pushkal and A.K. Shetty, "Detection of Human Papilloma Virus and Risk Factors among Patients with Head and Neck Squamous Cell Carcinoma Attending a 
Tertiary Referral Centre in South India", ASIAN PAC J CANCER P, 19(5), 1325, 2018. http://doi.org/10.22034/apjcp.2018.19.5.1325

[15] I. Akpolat, D.A. Smith, I. Ramzy, M. Chirala and D.R Mody, "The utility of p16INK4a and Ki-67 staining on cell blocks prepared from residual thin-layer cervicovaginal material”, CANCER CYTOPATHO,102(3),142-149, 2004. https://doi.org/10.1002/cncr.20258

[16] R.K. Sherwani, T. Khan, K. Akhtar, A. Zeba, F.A Siddiqui, K. Rahman and N. Afsan, "Conventional Pap smear and liquid based cytology for cervical cancer screening-A comparative study", J CYTOL, 24(4), 167, 2007. https://doi.org/10.4103/0970-9371.41888

[17] P. Basu, F. Meheus, Y. Chami, R. Hariprasad, F. Zhao and R. Sankaranarayanan, "Management algorithms for cervical cancer screening and precancer treatment for resource-limited settings", INT J GYNECOL OBSTET,138, 26-32, 2017. http://doi.org/10.1002/ijgo.12183

[18] H.Sakamoto, M. Takenaka, K. Ushimaru and T. Tanaka, "Use of LiquidBased Cytology (LBC) and cell blocks from cell remnants for cytologic, immunohistochemical, and immunocytochemical diagnosis of malignancy", OJPATHOLOGY,2(3), $58,2012$.

https://dx.doi.org10.4236/ojpathology.2012.23012. 